\title{
Increased furanocoumarin production by Glehnia littoralis roots induced via Agrobacterium rhizogenes infection
}

\author{
Masahiro Terato ${ }^{1}$, Aya Ishikawa ${ }^{1}$, Koji Yamada ${ }^{1}$, Yoshihiro Ozeki $^{2}$, Yoshie Kitamura ${ }^{3, *}$ \\ ${ }^{1}$ Graduate School of Biomedical Sciences, Nagasaki University, Nagasaki 852-8521, Japan; ${ }^{2}$ Faculty of Engineering, \\ Tokyo University of Agriculture and Technology, Koganei, Tokyo 184-8588, Japan; ${ }^{3}$ Faculty of Environmental Studies, \\ Nagasaki University, Nagasaki 852-8521, Japan \\ *E-mail: k-yoshie@nagasaki-u.ac.jp Tel \& Fax: +81-95-819-2759
}

Received February 18, 2011; accepted March 30, 2011 (Edited by T. Aoki)

\begin{abstract}
The objective of this study was to determine a possible application of hairy roots as biotechnological producers of furanocoumarins, by establishing root clones of Glehnia littoralis after infection from Agrobacterium rhizogenes. None of the root clones grew under hormone-free conditions, but when cultured in the presence of indole-3-butyric acid, growth varied substantially across clones. Analysis of the insertion and expression of pathogenic $\operatorname{rol}\left(A, B^{T L}, B^{T R}\right.$, and $\left.C\right)$ genes for 10 clones showed that the stable expression of $\mathrm{rolC}$ and irregular expression of $\mathrm{rolB}$ were detected in 2 clones, which exhibited active growth and higher furanocoumarin induction. Following ascorbic acid treatment, the productivity of xanthotoxin and bergapten in a root clone harbouring a pathogenic gene was 2.8 and 3.7 times greater than that recorded in wild-type roots, respectively. These results indicate that root cultures possessing pathogenic rol genes offer a potential means to produce furanocoumarins.
\end{abstract}

Key words: Agrobacterium rhizogenes, furanocoumarin induction, Glehnia littoralis, root clone.

Linear furanocoumarins, such as psoralen, bergapten, and xanthotoxin, are used clinically in the treatment of skin diseases such as leukoderma and psoriasis. The production of these compounds has traditionally been dependent on natural products, such as the fruits and juices of Citrus species. Recently, there have been several attempts to produce furanocoumarins by biotechnological means, using other plant species (Ekiert et al. 2001; Królicka et al. 2001; Sidwa-Gorycka et al. 2003; Sreelakshmi et al. 2007). For example, our research group has worked to produce furanocoumarins by using in vitro cells and organs of Glehnia littoralis subjected to elicitor treatment. However, the cell cultures from our study produced only bergapten, while leaf (but not root) cultures produced both bergapten and xanthotoxin following treatment with yeast extract (YE) (Ishikawa et al. 2007). Subsequent studies revealed that ascorbic acid (AsA) functioned as a powerful inducer of furanocoumarin production in organ cultures, especially in root cultures (Ishikawa et al. 2008). This result indicates the possible application of hairy roots as biotechnological producers of furanocoumarins, because ordinary hairy roots grow rapidly without phytohormones, in contrast to shoot cultures.

Hairy roots are induced by Agrobacterium rhizogenes infection. However, difficulty in obtaining transformants is often encountered with recalcitrant plants, which are often wild species. In such cases, the use of ampicillin for bacterial elimination is particularly helpful in establishing root clones, because ampicillin stimulates root elongation and facilitates the isolation of sterile roots (Rahman et al. 2004). Previously, we established root clones of G. littoralis using A. rhizogenes LBA9402, but none of the clones grew without ampicillin (Rahman et al. 2004). Hence, in the current study, 10 clones that had been randomly selected from 100 clones were maintained in $25 \mathrm{ml}$ of Murashige and Skoog (1962) (MS) basal medium supplemented with $5 \mathrm{mgl}^{-1}$ of indole-3-butyric acid (IBA) in a $100 \mathrm{ml}$ Erlenmeyer flask at $25^{\circ} \mathrm{C}$ and $80 \mathrm{rpm}$ in the dark, with subcultures every 6 weeks, as described for normal root cultures of $G$. littoralis (Ishikawa et al. 2007).

Since growth seemed to be variable among clones, growth was monitored visually throughout a time course: ca. $1 \mathrm{~cm}$ root tips from groups of 10 clones were cultured on solid MSIBA medium ( 9 tips per clone) together with

Abbreviations: AsA, ascorbic acid; BMT, bergaptol $O$-methyltransferase; CTAB, cetyltrimethylammonium bromide; DMSO, dimethyl sulfoxide; DW, dry weight; HPLC, high performance liquid chromatography; IBA, indole-3-butyric acid; MeJA, methyl jasmonate, MS, Murashige and Skoog; PAL, phenylalanine ammonia-lyase; RT-PCR, reverse transcription-polymerase chain reaction; SA, salicylic acid; UBI, ubiquitin; WT, wild type; YE, yeast extract.

This article can be found at http://www.jspcmb.jp/

Published online June 10, 2011 
a wild-type (WT) root for 6 weeks. The number and length of roots formed from each root tip was counted each week, and the weight was measured at 6 weeks. Root segments started to form a callus during the initial 2 weeks, although the extent of callus formation varied across clones. Thereafter, root differentiation from the callus began, followed by root elongation, leading to differing extents of root propagation in the clones. In brief, 3 clones (Gl2, Gl33, and Gl37) were rich in callus, resulting in higher weights than that recorded in other roots (Figure 1), but fewer roots were produced than WT (Figure 2A). However, one clone (G16) produced the

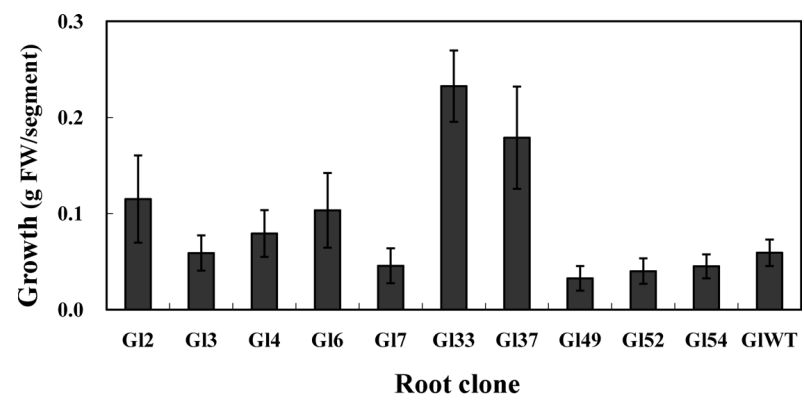

Figure 1. Different extents of root propagation (fresh weights basis) among 10 root clones and wild-type roots (WT). Root tips (ca. $1 \mathrm{~cm}$ in length) were cultured on solid MS medium containing $5 \mathrm{mg}^{-1}$ IBA for 6 weeks. Data are mean \pm sd $(n=9)$. highest number of roots $(31 \pm 5$ per segment) after 6 weeks, with a value almost double that of WT roots (16 \pm 4 per segment; Figure $2 \mathrm{~A})$. The number of roots formed by other clones, including G14, was similar to that recorded for WT roots. With respect to root length, Gl6 formed the longest root $(11.4 \pm 2.0 \mathrm{~mm})$, followed by G14 $(8.7 \pm 2.1 \mathrm{~mm})$, while other clones, including WT $(5.7 \pm 0.7 \mathrm{~mm})$, showed similar growth after 6 weeks (Figure 2B).

Since differences among clones appeared to be associated with the nature of the T-DNA insert present in each individual root clone, the insertion and expression of T-DNA was analysed by PCR and RT-PCR, respectively. There are 2 types of T-DNA on Ri-plasmids, depending on the strain of $A$. rhizogenes. Specifically, one type has 2 separate regions, namely $T L$ and $T R$, while the other type has only a single T-DNA region. Agrobacterium rhizogenes LBA9402 used for the infection of G. littoralis is known to be of the former type, with the presence of rolA to rolD on the $T L$-region, and the rolB gene homologue on the TR-DNA (rolB $\left.{ }^{T R}\right)$ (Bouchez and Camilleri 1990; White et al. 1985). However, with respect to hairy root induction, not all the transfer genes and associated functions have been determined yet, although $\operatorname{rol} A$, $\operatorname{rolB}$, and $\operatorname{rolC}$ (and especially rolB) are known to play crucial roles (Christey

\section{B}

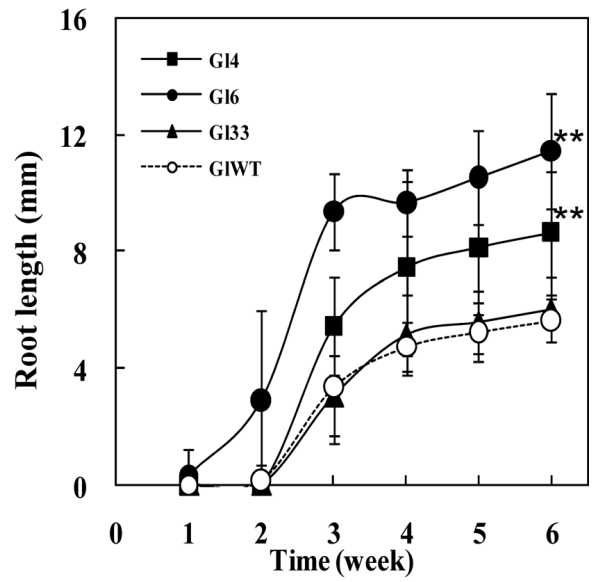

Figure 2. Time courses of lateral root formation (A) and increase in root length (B) by representative root clones and wild-type roots (WT). Root tips (ca. $1 \mathrm{~cm}$ in length) were cultured on solid MS medium containing $5 \mathrm{mg}^{-1} \mathrm{IBA}$ for 6 weeks. Root number and length of roots formed from root tips were counted every week. Data are mean $\pm s d(n=9)$. Data were analysed using Student's $t$-test. ** indicates the significant difference at the level of $p<0.01$ compared against WT.

Table 1. Gene-specific primers employed for PCR and RT-PCR

\begin{tabular}{|c|c|c|c|}
\hline Gene & Forward primer & Reverse primer & Product (bp) \\
\hline rolA & 5'-TGGAATTAGCCGGACTAAAC-3' & 5'-GCGTACGTTGTAATGTGTTG-3' & 660 \\
\hline $\operatorname{rolB}^{T L}$ & 5'-TAGCCGTGACTATAGCAAACCCCTCC-3' & 5'-GGCTTCTTTCTTCAGGTTTACTGCAG-3' & 670 \\
\hline $\mathrm{rolB} B^{T R}$ & 5'-ATCGCCATGGGCACATACGAAGGTC-3' & 5'-TTCGAGCAGGTTACGCCAGGAGG-3' & 673 \\
\hline rolc & 5'-TAACATGGCTGAAGACGACC-3' & 5'-AAACTTGCACTCGCCATGCC-3' & 534 \\
\hline GIPAL & 5'-GAAATCTTGAAACTCATGTCTAC-3' & 5'-CATCAATGGGTAGGTTGC-3' & 255 \\
\hline GlBMT & 5'-GTGTGGTTCATCTTGATGCT-3' & 5'-GTGGCCCTTATTGCATATTT-3' & 236 \\
\hline GlUBI & 5'-TCACCCTGGAGGTTGAGAG-3' & 5'-GACGCAACACAAGGTGAAGA-3' & 181 \\
\hline
\end{tabular}


2001). Therefore, $\operatorname{rol} A, \operatorname{rol} B^{T L}$, and rolC on $T L$ and $r o l B^{T R}$ on $T R$ were all examined in this report. Genomic DNA was isolated from fresh root materials by using cetyltrimethylammonium bromide (CTAB). Total RNA was extracted from ca. $50 \mathrm{mg}$ of fresh root material by using an RNeasy Plant Mini kit (Qiagen), according to the manufacturer's instructions. PCR and RT-PCR conditions are as described in a previous study by our research group (Rahman et al. 2004), except for the oligonucleotide primers, which are listed in Table 1.

PCR analysis showed that the bands corresponding to rolA, rolB ${ }^{T L}$, and rolC were detected in both G14 and Gl6. However, rolB ${ }^{T R}$ was found in G14 but not in Gl6 (Figure 3 ). Using these 2 clones, the expression of rol genes was examined by RT-PCR at various growth stages (1-3 weeks old) (Figure 4). The results showed that rolA expression was not detected at all, but rolC was always expressed. Unstable and irregular expression of $\mathrm{rolB}^{T L}$ in Gl6 and $\mathrm{rolB}^{T R}$ in G14, and no expression of $\mathrm{rolB}^{T L}$ in
G14 were found, providing a possible reason why IBA is required for growth.

To explore the variation in furanocoumarin production between different root clones, stress treatments were performed as described in a previous study (Ishikawa et al. 2008) with a minor modification. For stress treatments, $0.1 \mathrm{~g}$ of roots was inoculated into fresh medium ( $15 \mathrm{ml}$ in a $50 \mathrm{ml}$ flask). Then, after about 10 days, when new roots had started growing, the cultures were treated with various elicitors. In our previous work using WT, we found that only AsA was able to induce furanocoumarin production (Ishikawa et al. 2008). Therefore, we initially determined furanocoumarin productivity in clone G16, following treatments with potent elicitors, including AsA, YE, methyl jasmonate (MeJA), salicylic acid (SA), and hydrogen peroxide. YE (Difco Laboratories), autoclaved at $121^{\circ} \mathrm{C}$ for $15 \mathrm{~min}$, and MeJA (Sigma), dissolved in DMSO and filter sterilised, were added to produce $10 \mathrm{gl}^{-1}$ and $100 \mu \mathrm{M}$

Clone number

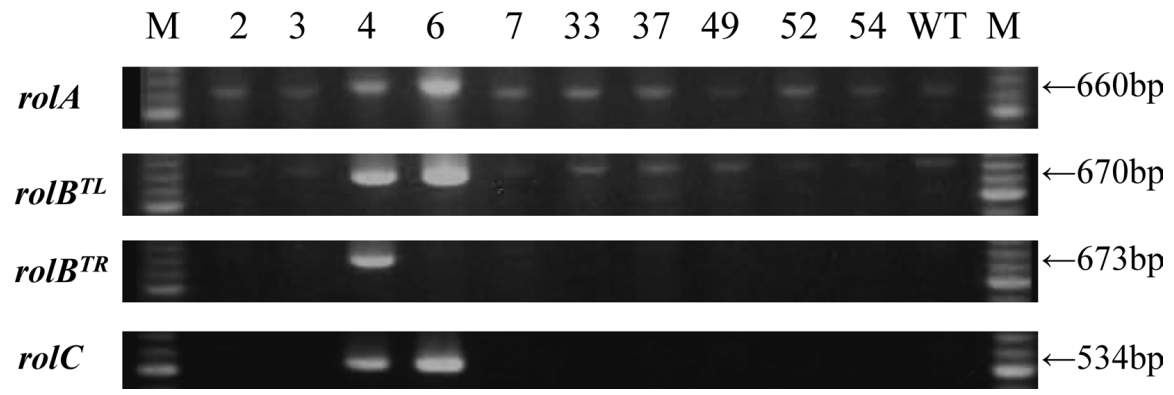

Figure 3. Analysis of $r o l$ gene insertion in 10 root clones and wild-type roots (WT). PCR was performed with rolA, rolB ${ }^{T L}$, rolB ${ }^{T R}$, and rolC specific primers for amplification of their specific fragments (see Table 1). Lane M, 100-bp molecular marker (larger band is 500 bp).

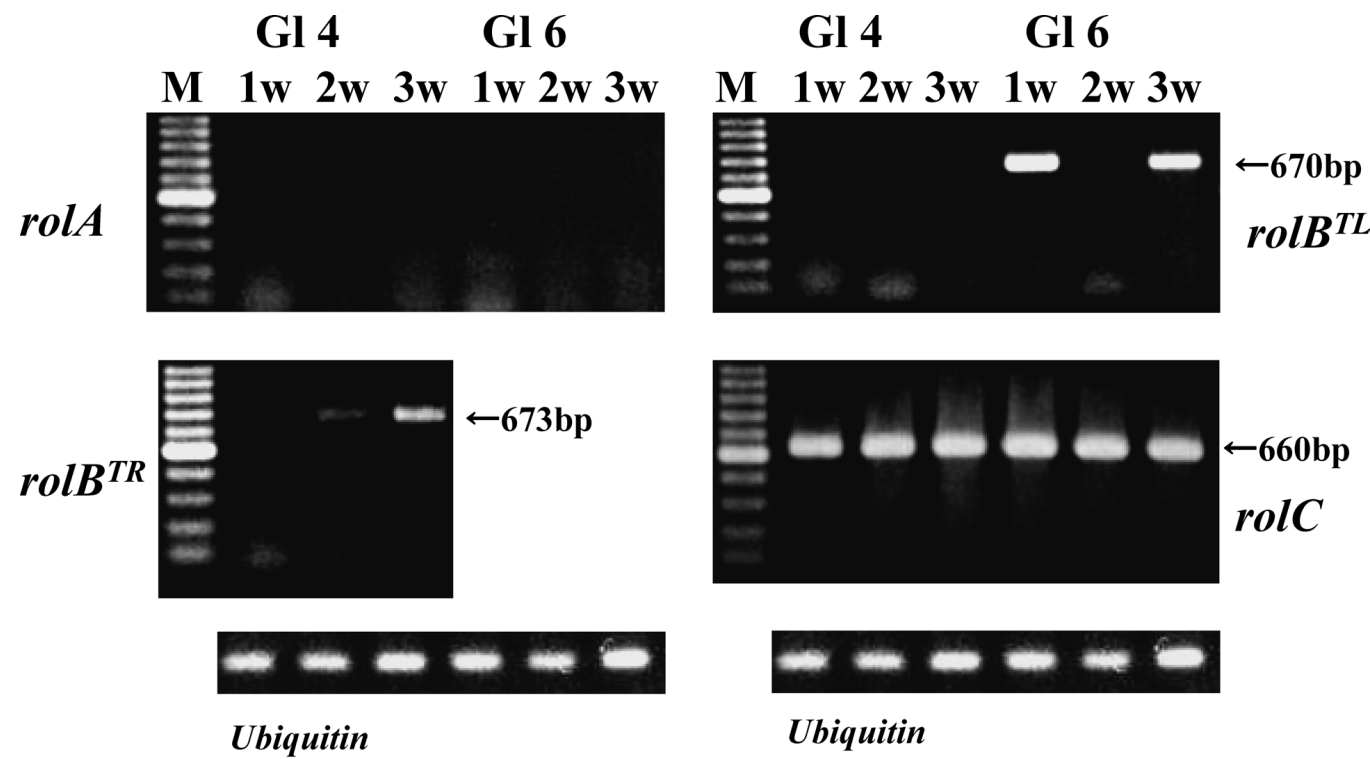

Figure 4. Analysis of rol gene expression in root clones G14 and Gl6. RT-PCR was performed with rolA, rolB ${ }^{T L}$, rolB ${ }^{T R}$, and rolC specific primers for amplification of their specific fragments (see Table 1). Ubiquitin (GlUBI) was used as a control. Lane M, 100-bp molecular marker (larger band is $500 \mathrm{bp})$. 
final concentrations, respectively. Aqueous solutions of AsA sodium salt, hydrogen peroxide, and SA, which were filter sterilised immediately before addition, were added to provide a final concentration of $20 \mathrm{mM}, 10 \mathrm{mM}$, and $100 \mu \mathrm{M}$, respectively. As controls, the cultures were treated with water or DMSO, as appropriate. Treatment was performed for $24 \mathrm{~h}$, and then the root cultures were harvested by vacuum filtration, to separate the roots from the medium. Furanocoumarins were extracted from cultured medium with ethyl acetate. 4-Methylumbelliferone $(5 \mathrm{mM}, 50 \mu \mathrm{l})$ was added to each sample, as an internal standard, before extraction. Furanocoumarins were separately quantified by HPLC, as described in previous work (Ishikawa et al. 2008).

The results showed that, in addition to AsA, YE and MeJA were also able to induce furanocoumarins, although induction was considerably inferior (Table 2). Since neither YE nor MeJA could induce furanocoumarin production in WT, as reported previously (Ishikawa et al. 2008), Gl6 was clearly more sensitive to these elicitors than WT. The time course of furanocoumarin production in Gl6 showed that both xanthotoxin and bergapten were detectable at $12 \mathrm{~h}$, and reached maxima at $24 \mathrm{~h}$ (data not shown). In all cases, the production of xanthotoxin was higher than that of bergapten. For the selection of high-producing clones for biotechnological application, the analysis of 10 clones treated for $24 \mathrm{~h}$ with $20 \mathrm{mM}$ AsA indicated that furanocoumarin production varied substantially, being dependent on the particular clone (Figure 5). G14 and Gl6, both of which possessed rol genes, showed higher
Table 2. Furanocoumarin production in response to potent elicitors

\begin{tabular}{lcc}
\hline \multirow{2}{*}{ Potent Elicitor } & \multicolumn{2}{c}{ Furanocomarin production $\left(\mu \mathrm{mol} \mathrm{g}^{-1} \mathrm{DW}\right)$} \\
\cline { 2 - 3 } & Xantotoxin & Bergapten \\
\hline MeJA & $0.36 \pm 0.04$ & $0.11 \pm 0.01$ \\
$\mathrm{DMSO}$ & n.d. & n.d. \\
$\mathrm{YE}$ & $0.21 \pm 0.03$ & $0.18 \pm 0.02$ \\
$\mathrm{H}_{2} \mathrm{O}_{2}$ & n.d. & n.d. \\
$\mathrm{SA}$ & n.d. & n.d. \\
$\mathrm{AsA}$ & $4.09 \pm 1.71$ & $1.53 \pm 0.76$ \\
$\mathrm{H}_{2} \mathrm{O}$ & n.d. & n.d.
\end{tabular}

Root cultures (clone G16) were treated with probable elicitors for $24 \mathrm{~h}$ and then the culture medium was analyzed for furanocoumarin content. MeJA, methyl jasmonate; DMSO, dimethyl sulfoxide; YE, yeast extract; SA, salicylic acid; AsA, ascorbic acid; n.d., not detected; Dw, dry weight. MeJA was dissolved in DMSO, while other solutions were dissolved in water. Data are means \pm sd of triplicates. Dw, dry weight.

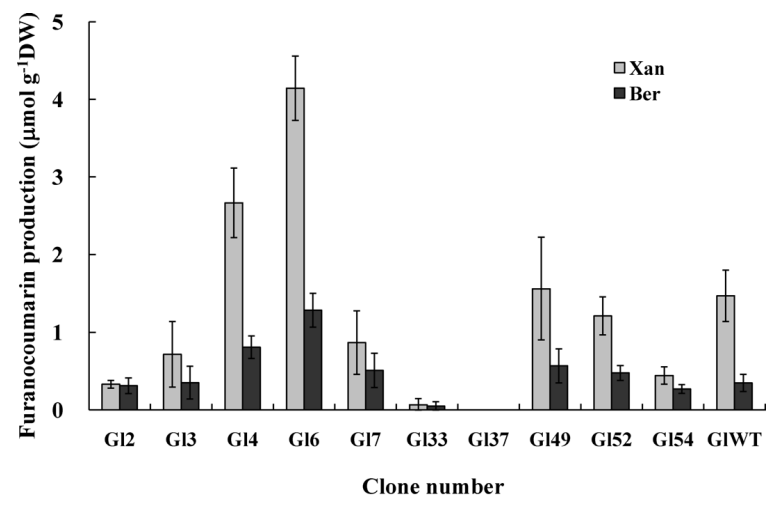

Figure 5. Production of xanthotoxin and bergapten from 10 root clones and wild-type roots. Roots (ca. $0.1 \mathrm{~g}$ ) were precultured in liquid medium ( $15 \mathrm{ml}$ in a $50 \mathrm{ml}$ flask) and then 2-week-old roots were treated with $20 \mathrm{mM}$ AsA for $24 \mathrm{~h}$. Data are mean $\pm \mathrm{sd}(\mathrm{n}=4)$. Dw, dry weight.
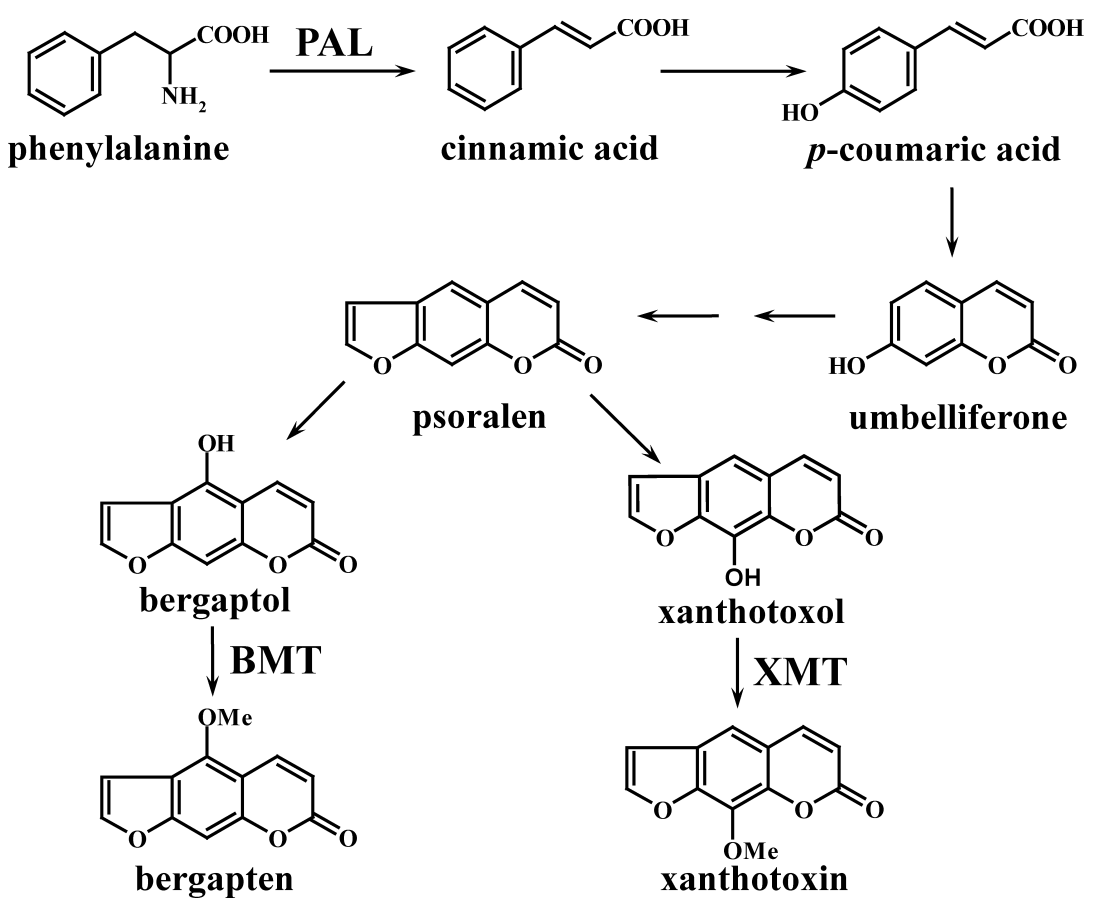

Figure 6. Biosynthetic pathway leading to furanocoumarins. PAL, phenylalanine ammonia-lyase; BMT, bergaptol $O$-methyltransferase; XMT, xanthotoxol $O$-methyltransferase. 


\section{$-\infty-P A L$ $B M T$

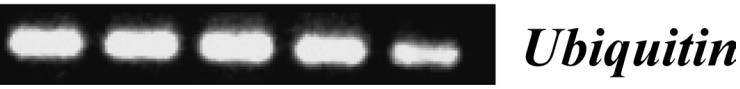 \\ $\begin{array}{llllll}0 & 6 & 9 & 12 & 24 & (h)\end{array}$}

Figure 7. Time courses of gene expression of PAL and BMT in $G$. littoralis roots supplied with $20 \mathrm{mM}$ AsA. RT-PCR was performed with GIPAL, GIBMT, and GIUBI specific primers for amplification of specific fragments (see Table 1). Ubiquitin (GlUBI) was used as a control.

productivity, whereas Gl37 and Gl33 produced few or no furanocoumarins. The most promising clone, Gl6, produced 2.8 times more xanthotoxin $(4.14 \pm 0.42 \mu \mathrm{mol}$ $\left.\mathrm{g}^{-1} \mathrm{DW}\right)$ and 3.7 times more bergapten $(1.29 \pm 0.22$ $\left.\mu \mathrm{mol} \mathrm{g}^{-1} \mathrm{DW}\right)$ than WT $(1.47 \pm 0.33$ and $0.35 \pm 0.11$ $\mu \mathrm{mol} \mathrm{g}^{-1} \mathrm{DW}$, respectively).

To confirm de novo induction of furanocoumarin in transformed roots, we determined the time course of gene expression for phenylalanine ammonia-lyase (PAL) and for bergaptol $O$-methyltransferase (BMT) in Gl6 after AsA treatment. PAL catalyses the first step of the phenylpropanoid pathway and is an elicitation marker, while BMT catalyses the final step of bergapten biosynthesis (Figure 6). GlBMT, GlPAL, and GlUBI (GenBank accession numbers AB363638, AB374257, and $\mathrm{AB} 363637$, respectively) were cloned and sequenced in a previous study by our research group (Ishikawa et al. 2009). Specific primers used for RT-PCR are listed in Table 1. The gene expression of xanthotoxol $O$ methyltransferase was not determined because of its current unavailability, although xanthotoxin production by root cultures was higher than that of bergapten. The result showed that GIBMT and GIPAL were clearly induced after $6 \mathrm{~h}$ of elicitation, and became maximal after around 9-12h (Figure 7). Since furanocoumarin was detected after $12 \mathrm{~h}$, and became maximal after $24 \mathrm{~h}$, de novo induction of furanocoumarin in root cultures by elicitation was confirmed.

In this paper, we reported that all clones of G. littoralis root cultures, which were obtained following $A$. rhizogenes infection, lacked growth without the hormone, but that growth behaviour was altered when cultured with IBA. Two superior clones harbouring bacterial genes showed enhanced sensitivity to elicitors, resulting in increased de novo synthesis of xanthotoxin and bergapten.

\section{Acknowledgements}

We thank Dr Nicholas Walton (UK) for editing the English text. This work was supported in part by a Grant-in-Aid (C, 20580279) from the Japan Society for the Promotion of Science.

\section{References}

Bouchez D, Camilleri C (1990) Identification of putative rol B gene on the TR-DNA of the Agrobacterium rhizogenes A4 Ri plasmid. Plant Mol Biol 14: 617-619

Christey MC (2001) Use of Ri-mediated transformation for production of transgenic plants. In Vitro Cell Dev Biol-Plant 37: $687-700$

Ekiert H, Chołoniewska M, Gomólka E (2001) Accumulation of furanocoumarins in Ruta graveolens L. shoot culture. Biotechnol Lett 23: 543-545

Ishikawa A, Kitamura Y, Ozeki Y, Watanabe M (2007) Different responses of shoot and root cultures of Glehnia littoralis to yeast extract. $J$ Nat Med 61: 30-37

Ishikawa A, Kobayashi N, Kitamura Y (2008) Ascorbic acid induces furanocoumarin production in organ cultures of Glehnia littoralis. Planta Med 74: 1517-1519

Ishikawa A, Kuma T, Sasaki H, Sasaki N, Ozeki Y, Kobayashi N, Kitamura Y, (2009) Constitutive expression of bergaptol $O$ methyltransferase in Glehnia littoralis. Plant Cell Rep 28: 257-265

Królicka A, Staniszewska I, Bielawski K, Maliński E, Szafranek J, Łojkowska E (2001) Establishment of hairy root cultures of Ammi majus. Plant Sci 160: 259-264

Murashige T, Skoog F (1962) A revised medium for rapid growth and bioassay with tobacco tissue cultures. Physiol Plant 15: 473-497

Rahman L, Ikenaga T, Kitamura Y (2004) Penicillin derivatives induces chemical structure-dependent root development, and application for plant transformation. Plant Cell Rep 22: 668-677

Sidwa-Gorycka M, Królicka A, Kozyra M, Głowniak K, Bourgaud F, Łojkowska E (2003) Establishment of a co-culture of Ammi majus L. and Ruta graveolens L. for the synthesis of furanocoumarins. Plant Sci 165: 1315-1319

Sreelakshmi L, Kishor PBK, Reddy KJ (2007) Enhanced production of psoralen, a furanocoumarin in callus cultures of Cullen corylifolium (L.) Medik. Plant Cell Biotechnol Mol Biol 8: 27-32

White FF, Taylor BH, Huffman GA, Gordon MP, Nester EW (1985) Molecular and genetic analysis of the transferred DNA regions of the root-inducing plasmid of Agrobacterium rhizogenes. J Bacteriol 164: 33-44 